\title{
LONG-TERM SOLAR FLUX OBSERVATIONS WITH IRKUTSK INCOHERENT SCATTER RADAR (IISR) IN 2011-2019
}

\author{
A.G. Setov \\ Institute of Solar-Terrestrial Physics SB RAS, \\ Irkutsk, Russia,setov@iszf.irk.ru \\ D.S. Kushnarev \\ Institute of Solar-Terrestrial Physics SB RAS, \\ Irkutsk, Russia,ds_k@iszf.irk.ru
}

\author{
R.V. Vasilyev \\ Institute of Solar-Terrestrial Physics SB RAS, \\ Irkutsk, Russia,roman_vasilyev@iszf.irk.ru \\ A.V. Medvedev \\ Institute of Solar-Terrestrial Physics SB RAS, \\ Irkutsk,Russia,medvedev@iszf.irk.ru
}

\begin{abstract}
Irkutsk incoherent scatter radar (IISR) is an oblongish horn antenna that operates in a meter waveband $(154-162 \mathrm{MHz})$, has a $0.5^{\circ} \times 20^{\circ}$ beam, and a frequency steering allowing us to tilt the beam by $30^{\circ}$ to the south. Besides active measurements of ionospheric conditions and monitoring of space objects, the radar is regularly used for passive radio astronomical observations. From May to August, the Sun crosses the radar field of view and can be in the maximum of the radiation pattern for about two hours. The known shape of the radiation pattern and the high sensitivity of the receiver allow us to conduct calibrated measurements of the solar flux in solar flux units during this period. We have developed a new approach to the calibration, which can be applied to all IISR archival passive data. In the paper, we present long-term observations (2011-
\end{abstract}

2019) of the solar flux in May and summer. We describe the measurement method, present daily average values of the solar flux for this period of passive measurements, and compare it with the solar activity $F 10.7$ index and solar flux measurements made at the Australian observatory Learmonth at $245 \mathrm{MHz}$. We show that the daily average flux for the period of observations at a frequency of $\sim 161 \mathrm{MHz}$ generally has values from 5 to $10 \mathrm{sfu}$.

Keywords: solar flux, IISR, calibration, F10.7, Learmonth.

\section{INTRODUCTION}

Activity on the Sun's surface, especially in the solar corona, is largely responsible for space weather conditions. During solar flares, X-ray and EUV emission increases sharply, thus causing an increase in ionization and disturbances in Earth's ionosphere [Tsurutani et al., 2009]. Coronal holes and streamers are sources of the fast solar wind, which triggers geomagnetic disturbances [Zirker, 1977]. Coronal mass ejections cause an increase in solar wind density and velocity and highenergy particle ejections, thus generating geomagnetic storms (e.g., [Zhang et al., 2007]). The solar corona can be monitored by radio emission in the meter waveband since its sources, located closer to the solar surface, are shielded by the plasma [Shibasaki et al., 2011]. Besides the constant solar emission in VHF, there are bursts representing both short-term and long-term increases in radio emission.

Interest in VHF solar radio emission has led to the advent of many spectrometers, which typically have an antenna with a small aperture and a wide radiation pattern, such as CALLISTO spectrometers, the Irkutsk spectropolarimeter SSMD with log-periodic antennas [Benz et al., 2005; Muratova et al., 2019], or the spectrograph HiRAS with a log-periodic antenna and two parabolic antennas 10 and $6 \mathrm{~m}$ in diameter [Kondo et al., 1995]. While these instruments can examine the behavior of the spectrum of intense bursts, they have low sensitivity and are rarely calibrated, therefore cannot determine the solar flux value and measure the background solar radio emission. Calibrated, highly sensitive measurements of the spectral flux density of solar emission or just of the solar flux measured in sfu (solar flux units; $1 \mathrm{sfu}=10^{-22} \mathrm{~W} \cdot \mathrm{m}^{-2} \cdot \mathrm{Hz}^{-1}$ ) are therefore particularly valued. Nowadays there are few instruments capable of making such measurements (e.g., ARTEMIS-IV [Kontogeorgos et al., 2006], AMATERAS [Iwai el al., 2012]), or the measurements are performed only from time to time. So, The Nancay radioheliograph works in a range of 150 to $450 \mathrm{MHz}$, but data is available only before 2015 . The large radio telescopes LOFAR (10-230 MHz) and MWA (70-300 MHz) are sometimes used for solar observations, but they have many other research tasks. Regularly calibrated measurements of solar flux at eight discrete frequencies (245, 410, 610, 1415, 2696, 4995, 8800, and $15400 \mathrm{MHz}$ ) from the Radio Solar Telescope Network (RSTN) of the United States Air Force are available starting from 1966 [Giersch et al., 2017]. The network includes multiple stations deployed at different times, some of which have operated to date. Frequencies were selected to be close to operating frequencies of different radioelectronic devices and antennas used in civil and military purposes, but only the frequency of $245 \mathrm{MHz}$ falls within the meter waveband.

Irkutsk Incoherent Scatter Radar (IISR) was originally a military radar, which was modified for research purposes [Potekhin et al., 2009]. It operates in a 154$162 \mathrm{MHz}$ frequency band $(\lambda=\sim 1.9 \mathrm{~m})$ and has an oblongish two-channel horn antenna of $246 \times 12 \mathrm{~m}$, which provides two $0.5^{\circ} \times 20^{\circ}$ beams. Due to the large antenna 
and high power, the radar is used for ionospheric sounding through incoherent scattering. When IISR does not make active measurements, it detects radioastronomical signals, including signals from the Sun in the period from May to August. Due to the narrow beam, known shape of the radiation pattern, and high sensitivity, we can calibrate the IISR receiver to obtain solar flux values. The paper describes the method of measuring solar flux and reports observational results received from 2011 to 2019.

One of the main solar activity indices in space weather research is the $F 10.7$ index - solar flux at a wavelength of $10.7 \mathrm{~cm}(\sim 2.8 \mathrm{MHz})$ [Tapping, 2013; Bilitza, 2000; Verbanac, 2010]. However, although the F10.7 index correlates well with solar activity, it covers only a part of the processes occurring on the Sun. We compare IISR daily average solar flux, the solar activity index $F 10.7$, and the flux recorded at Learmonth Solar Observatory of the RSTN network at a frequency of $245 \mathrm{MHz}$.

\section{MEASUREMENT TECHNIQUE}

IISR has a frequency steering: the radar beam is controlled electronically, and the operation direction is determined by frequency. In transmission or reception at a frequency of $154 \mathrm{MHz}$, the beam is vertical; at a frequency of $162 \mathrm{MHz}$, it is tilted by $30^{\circ}$. IISR has made passive observations for about ten years, and over this period, the way that radioastronomical signals are processed has been changed several times. Initially, for the data on 2011-2014, measurements were made at over 80 discrete frequencies covering the operating frequency range [Vasilyev et al., 2013]. Sequential switching to a new frequency, signal reception and digitization, which yielded from 1024 to 4096 complex points depending on settings, were performed. The receiver had a bandwidth of $25 \mathrm{kHz}$. Received signals were used to determine averaged power and coherence coefficient. In 2015 , the receiver was modernized to receive signals with a bandwidth of $700 \mathrm{kHz}$. If we take the Fourier spectrum of such a signal, each individual frequency of the spectrum will correspond to a particular beam direction in space. As a result, the number of central frequencies used to receive signals was reduced to 11 . Since 2015, the archive has stored recorded quadratures rather than averaged parameters, which allows us to choose a processing method more flexibly. In 2017, the reception mode was again changed - it was necessary to increase time resolution to monitor powerful radio sources, such as the Sun, Cygnus-A, Crab Nebula, and Cassiopeia-A. Currently, passive measurements during the day are made in two modes: tracking of radio sources at one central frequency, which varies so that a source would be always at the radiation pattern maximum [Vasilyev et al., 2016], and scanning at 11 central frequencies covering the entire IISR frequency range.

Over the years of active and passive observations, the IISR receiver has constantly been modified and its characteristics have been changed, therefore it has to be routinely calibrated during processing of archival passive data. The advantage of IISR over many other instruments is the known shape of its radiation pattern, which is necessary for precise positioning and calibration. We have previously proposed a method for calibrating and determining the solar flux value, which is based on the signal from Cygnus-A in the tracking mode, for data after 2017 [Setov et al., 2018]. This method cannot be applied to the data before 2017, therefore in this paper we have chosen another approach and utilize the sky noise recorded in the scan mode as a calibration signal. There are a variety of calibrated measurements of the sky noise at different frequencies, carried out with precision radio telescopes and dedicated instruments. The Global Sky Model (GSM) [de Oliveira-Costa et al., 2008] combines sky noise maps and is able to interpolate the sky brightness temperature distribution in frequencies from $10 \mathrm{MHz}$ to $100 \mathrm{GHz}$. We have used this model to create sky noise maps at a frequency of $158 \mathrm{MHz}$ and have performed convolution with the radar's radiation pattern at different inclinations of the main beam. Since the data is obtained in the scan mode every day, we can compare the observed and model values of sky noise power by first removing all discrete sources as poorly approximated by GSM from consideration. This allows us to find daily calibration coefficients of the receiver - gain and receiver noise of each channel. The advantage of this approach is that the scan mode covers all operating frequencies of the radar, hence the receiver is calibrated at all possible frequencies. This is important as the IISR antenna and receiver itself have an irregular frequency response.

We should also mention the filtration method for passive data. Near IISR is an active station operating in the same frequency range, so in many quadratures recorded after 2017 is high-amplitude interference. We have tested several filtration methods, and the most effective proved to be the threshold median filter with median deviation when the deviation is estimated using the median rather than the mean. In this case, the estimated deviation is more robust to intensive outliers in the data. We have also employed this filter to determine bursts in the solar flux values obtained, as well as to filter point sources in sky noise calibration.

Since angular dimensions of the main beam are $0.5^{\circ} \times 20^{\circ}$, which is comparable to the angular dimensions of the visible solar disk, defining the solar flux requires an understanding of the Sun's brightness distribution across the disk. As a brightness distribution model we use a Gaussian-smoothed ellipse, which is based on previous solar observations [Leblanc, le Squeren, 1969]. The solar flux method is described in more detail in [Setov et al., 2018]. Nonetheless, when a burst occurs on the Sun, the brightness distribution model no longer shows actual distribution. We have simulated an error in determining the solar flux in this case by adding a Gaussian-shaped burst with different intensity and deviation from the solar equator. Figure 1 shows the results of the error simulation, where along the $\mathrm{X}$-axis is the deviation from the solar equator (deviation along the equator makes no significant changes), and along the $\mathrm{Y}$-axis is the ratio of the added burst flux to the total background solar flux. The error can be significant if the burst flux exceeds the background solar flux. Contrary to expectations, the error decreases if 


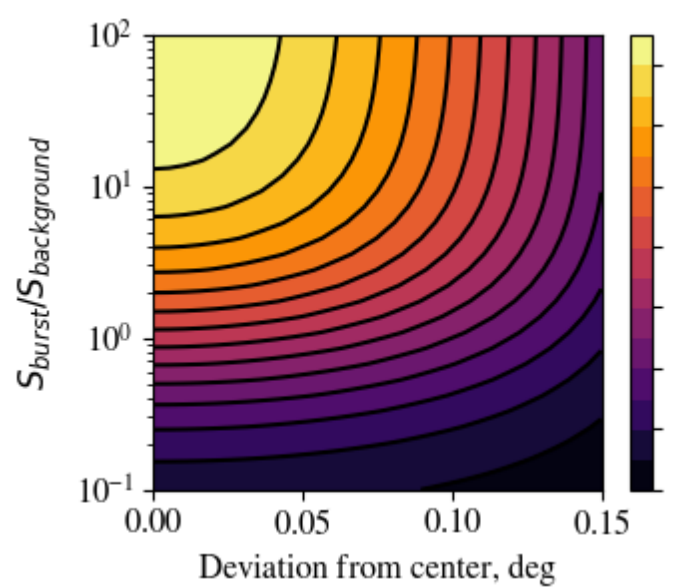

Figure 1. Relative error in identifying the solar flux at the occurrence of a burst on the Sun

the burst occurs farther from the equator. This is due to the fact that the farther is the burst, the farther it is from the center of the radiation pattern, and hence the smaller is the contribution it makes to the total flux.

IISR can receive a solar flux with a resolution of a few seconds for two or three hours, depending on the season. To focus on the long-term solar flux observation, the daily average solar flux is calculated as the average of all the flux values measured during the day at the frequency at which the radiation pattern maximum is aimed at the solar disk center. The frequency is changed during the day due to the changing position of the Sun, covering the range $159-161.5 \mathrm{MHz}$ in the middle of the summer and 161-162.5 MHz in spring and late summer. Since the frequency is changed slightly, we assign measurements of the daily average solar flux to the frequency of $\sim 161 \mathrm{MHz}$. Intense radio emission bursts can make a significant shift in the estimated daily average flux, so data is pre-filtered. From daily time series we remove the flux values having a level five times higher than the median value in the 15 min observation window. Thus, when calculating the daily average flux, we ignore short-term intense bursts lasting for several tens of seconds, but still take into account long-term variations in the flux occurring, for example, during noise storms. Also noteworthy is that the IISR antenna includes a polarization filter, so we receive only a part of the total flux. To determine the total flux, the received power is doubled, and, if it is known that the solar radio emission is polarized in VHF (e.g., during noise storms [Dulk et al., 1984] or type IV bursts [Liu et al., 2018]), the data should be treated with caution.

\section{LONG SERIES OF SOLAR FLUX MEASUREMENTS}

Figure 2 presents IISR measurements of the daily average solar flux for 2011-2013, 2015, 2017-2019 from early May to mid-August, as well as the solar flux at $245 \mathrm{MHz}$ received at Learmonth Solar Observatory, and the F10.7 flux. In 2014 and 2016, IISR did not make passive measurements during solar observation. In 2015, measurements were performed only for two weeks in late June - early July. Data gaps also appear during active ionospheric observations made with IISR, for example, in June-July 2012, 2013, and 2019. For clarity, there are two scales in Figure 2: left - for IISR and Learmonth data, right — for the $F 10.7$ flux, as these measurements have different scales of values. There are gaps in the IISR and Learmonth data, while the $F 10.7$ flux is measured almost continuously. The IISR data for some days was excluded from consideration because of poor calibration, strong interference, or high solar activity (due to the above limitations of the solar flux method).

The IISR solar flux is seen to be in most cases lower than the Learmonth one. As expected, under quiet conditions the flux is higher at a higher frequency. The IISR and Learmonth fluxes are in close agreement quiet and disturbed periods almost exactly coincide. A simultaneous increase in the VHF flux is seen to occur during high solar activity, for example in early August 2011, late June 2015, and mid-July 2017. Only in a few cases, the daily average flux at $\sim 161 \mathrm{MHz}$ is greater than that at $245 \mathrm{MHz}$. The flux values exhibit periods of low and higher solar activity: in 2012-2015 the flux is higher at all of the three frequencies than in 2017-2019. In addition, in those years there were more events during which the daily average flux increased for several days. The $F 10.7$ flux is in much poorer agreement with both IISR data and Learmonth data. We can identify periods when under a small change the $F 0.7$ flux in VHF increased several times, for example, in August 2011 and July 2017. Particularly noteworthy among the periods when the disturbance occurred in all the ranges are July 2012 and late June 2015.

To examine the distribution of the IISR daily average solar flux for that period, we have built a histogram (Figure 3) for values up to $100 \mathrm{sfu}$. Figure 2 shows that only in 2018-2019 on observation days there were no disturbances, so the distribution is not typical of the quiet Sun and is shifted toward higher values. The solar flux at $\sim 161 \mathrm{MHz}$ generally varies from 5 to $10 \mathrm{sfu}$ with a maximum at $8 \mathrm{sfu}$. This result is consistent with those obtained previously in [Leblanc, le Squeren, 1969]. The daily average flux values above $100 \mathrm{sfu}$ were observed only in two cases during the noise storm in July 2017, when the flux was as high as $500 \mathrm{sfu}$. It should again be noted that we do not consider bursts whose intensity may be well over $100 \mathrm{sfu}$ [Giersch et al., 2017].

\section{CONCLUSION}

IISR has long been used in the passive mode of observation of radio sources. In the period from May to August, it is possible to identify the solar flux at a frequency of $\sim 161 \mathrm{MHz}$, which is practically not affected by other calibrated instruments. We have developed a new approach to calibration of passive measurements at IISR, applicable to all archival data and based on GSM, which approximates sky noise maps.

The new approach has enabled us to calibrate the receiver in the entire operating frequency range and to measure the solar flux. We have found that the daily average solar flux at this frequency generally ranges from 5 to $10 \mathrm{sfu}$. Data on the daily average solar flux measured with IISR agrees well with measurements at $245 \mathrm{MHz}$ 


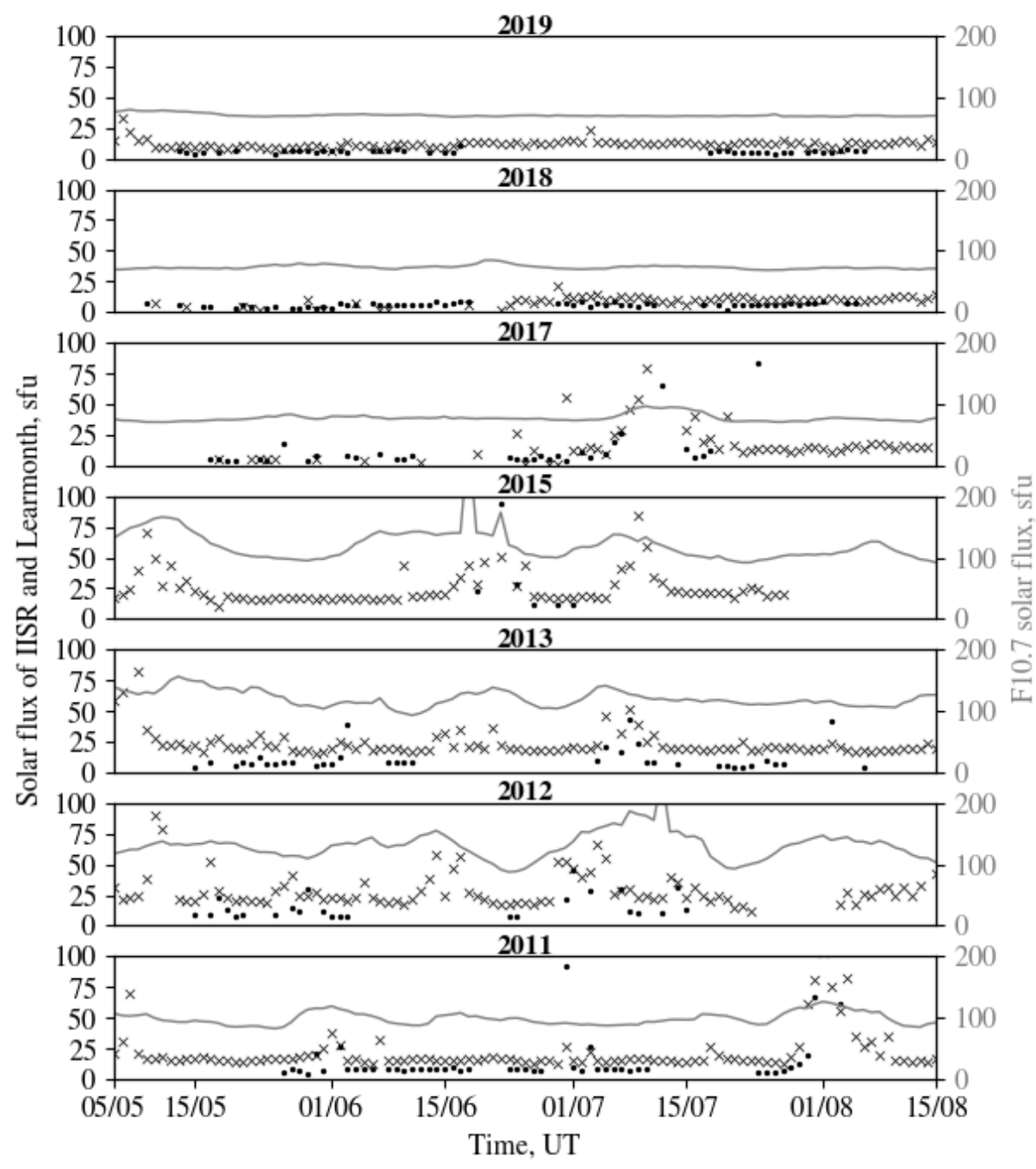

Figure 2. Solar flux measurements in sfu with IISR from 2011 to 2019 from May 5 to August 15. Black dots indicate the daily average flux at frequencies around $161 \mathrm{MHz}$, obtained with IISR. Crosses mark the average flux at $245 \mathrm{MHz}$, received at Learmonth Solar Observatory; the solid line is the $F 10.7$ index (solar flux at a frequency of $\sim 2.8 \mathrm{GHz}$ ). The left scale is the solar flux value in sfu for IISR and Learmonth data; the right scale, for $F 10.7$

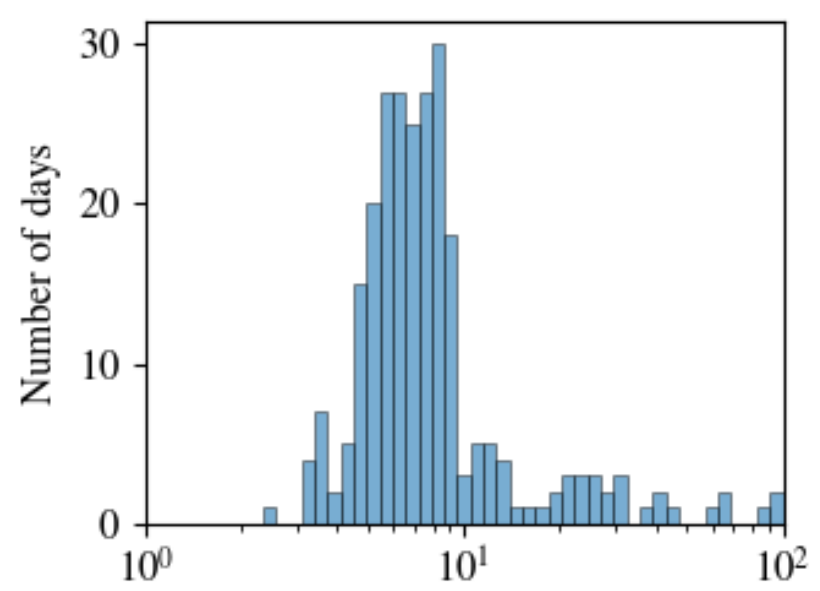

Solar flux, sfu

Figure 3. Histogram of IISR measurements of the daily average solar flux from 2011 to 2019 from Learmonth Solar Observatory. The flux at $\sim 161$ $\mathrm{MHz}$ is predictably lower than that at $245 \mathrm{MHz}$ when there are no disturbances on the Sun. On the other hand, dynamics of the F10.7 flux differs from that of the VHF flux. This again suggests that when analyzing solar activity it is insufficient to use only one index as it would not reflect all processes occurring on the Sun.

IISR solar observations have a high time resolution of $\sim 1 \mathrm{~s}$, which allows us to study the fine dynamics structure of processes on the Sun. In the future, the method for making calibrated solar flux measurements with IISR will be improved for in-depth study of bursts and noise storms.

The work was supported by the Russian Science Foundation (project No. 18-17-00042). Observations were obtained using the equipment of Center for Common Use «Angara» [http://ckp-rf.ru/ckp/3056/] and with budgetary funding of Basic Research program II.12. 
Solar flux data obtained at Learmonth Solar Observatory is available at the ftp server of Australian Government Bureau of Meteorology [ftp://ftpout.sws.bom.gov.au].

\section{REFERENCES}

Benz A.O., Monstein C., Meyer H. CALLISTO - A new concept for solar radio spectrometers. Solar Phys. 2005, vol. 226. pp. 143-151. DOI: 10.1007/s11207-005-5688-9.

Bilitza D. The importance of EUV indices for the International Reference Ionosphere. Phys. Chem. Earth (C). 2000, vol. 25. no. 5-6, pp. 515-521. DOI: 10.1016/S14641917(00)00068-4.

de Oliveira-Costa A., Tegmark M., Gaensler B.M., Jonas J., Landecker T.L, Reich P. A model of diffuse galactic radio emission from $10 \mathrm{MHz}$ to $100 \mathrm{GHz}$. Mon. Not. R. Astron. Soc. 2008, vol. 388 . pp. 247-260. DOI: 10.1111/j.13652966.2008.13376.x.

Dulk G.A., Suzuki S., Sheridan K.V. Solar noise storms: the polarization of storm Type III and related bursts. Astron. Astrophys. 1984, vol. 130, pp. 39-45.

Giersch O.D., Kennewell J. and Lynch M. Solar radio burst statistics and implications for space weather effects. Space Weather. 2017, vol. 15. no. 11. pp. 1511-1522. DOI: 10.1002/2017SW001658.

Iwai K., Tsuchiya F., Morioka A., Misawa H. IPRT/ AMATERAS: A new metric spectrum observation system for solar radio bursts. Solar Phys. 2012, vol. 277. pp. 447-457. DOI: 10.1007/s11207-011-9919-y.

Kondo T., Isobe T., Igi S., Watari S., Tokumaru M. Hiraiso Radio Spectrograph (HiRAS) for monitoring solar radio bursts. J. Communications Research Laboratory. 1995, vol. 42. no. 1 , pp. 111-119.

Kontogeorgos A., Tsitsipis P., Caroubalos C., Moussas X., Preka-Papadema P., Hilaris A., et al. The improved ARTEMIS IV multichannel solar radio spectrograph of the University of Athens. Experimental Astronomy. 2006, vol. 21. pp. 41-55. DOI: $10.1007 / \mathrm{s} 10686-006-9066-\mathrm{x}$.

Leblanc Y., Le Squeren A.M. Dimensions, Temperature and electron density of the quiet corona. Their variations during the solar cycle. Astron. Astrophys. 1969, vol. 1. pp. 239 248.

Liu H., Chen Y., Cho K., Feng S., Vasanth V., Koval A., et al. A solar stationary type IV radio burst and its radiation mechanism. Solar Phys. 2018, vol. 293. pp. 58. DOI: 10.1007/s11207-018-1280-y.

Muratova N.O., Muratov A.A., Kashapova L.K. Results of work of new spectropolarimeter for solar radio emission observations in the range 50-500 MHz. Solar-Terr. Phys. 2019, vol. 5. no. 3, pp. 3-9. DOI: 10.12737/stp-53201901.

Potekhin A.P., Medvedev A.V., Zavorin A.V., Kushnarev D.S., Lebedev V.P, Lepetaev V.V., Shpynev B.G. Recording and control digital systems of the Irkutsk Incoherent Scatter Radar. Geomagnetism and Aeronomy. 2009, vol. 49. no. 7, pp. 1011-1021. DOI: 10.1134/S0016793209070299.

Setov A.G., Globa M.V., Medvedev A.V., Vasilyev R.V., Kushnarev D.S. First results of absolute measurements of solar flux at the Irkutsk Incoherent Scatter Radar (IISR). Solar-Terr. Phys. 2018, vol. 4. no. 3, pp. 24-27. DOI: $10.12737 /$ stp-43201804.

Shibasaki K., Alissandrakis C.E., Pohjolainen S. Radio emission of the quiet Sun and active regions (Invited Review). Solar Phys. 2011, vol. 273. pp. 309-337. DOI: 10.1007/ s11207-011-9788-4.

Tapping K.F. The $10.7 \mathrm{~cm}$ solar radio flux (F10.7). Space Weather. 2013, vol. 11. pp. 394-406. DOI: 10.1002/swe.20064.
Tsurutani B.T., Verkhoglyadova O.P., Mannucci A.J., Lakhina G.S, Li. G., Zank G.P. A brief review of "solar flare effects" on the ionosphere. Radio Sci. 2009, vol. 44. no. RS0A17. DOI: 10.1029/2008RS004029.

Vasilyev R.V., Globa M.V., Kushnarev D.S., Lebedev V.P., Medvedev A.V., Ratovsky K.G. Model of discrete cosmic radiosource signal for Irkutsk incoherent scatter radar. XXV Vserossijskaya otkrytaya konferentsiya «Rasprostranenie radiovoln» [XXV All-Russian Open Conference "Propagation of Radio Waves"]. Tomsk, 2016, vol. 3, pp. 122-125. (In Russian).

Vasilyev R.V., Kushnarev D.S., Kashapova L.K., Lebedev V.P., Medvedev A.V., Nevedimov N.I., Ratovsky K.G. First results of radio observations of the Sun and powerful discrete sources at Irkutsk Radar. Astronomicheskii zhurnal [Astron. Rep.], 2013, vol. 90. no. 11, pp. 948-958. (In Russian). DOI: 10.7868/S0004629913110078.

Verbanac G., Vršnak B., Temmer M., Mandea M., Korte M. Four decades of geomagnetic and solar activity: 1960-2001. J. Atm. Solar-Terr. Phys. 2010, vol. 72. pp. 607-616. DOI: 10.1016/j.jastp.2010.02.017.

Zhang J., Richardson I.G., Webb D.F. et al. Solar and interplanetary sources of major geomagnetic storms $\left(\Gamma^{\mathrm{at}}\right.$ < -100 nT) during 1996-2005. J. Geophys. Res. 2007, 112. no. A10102. DOI: 10.1029/2007JA012321.

Zirker J.B. Coronal holes and high-speed wind stre Rev. Geophys. Space Phys. 1977, vol. 15. no. 3, pp. 257-269. DOI: 10.1029/RG015i003p00257.

URL: http://ckp-rf.ru/ckp/3056/ (accessed June 27, 2020).

URL: ftp://ftp-out.sws.bom.gov.au (accessed June 27, 2020).

How to cite this article

Setov A.G., Kushnarev D.S., Vasilyev R.V., Medvedev A.V. Long-term solar flux observations with Irkutsk Incoherent Scatter Radar (IISR) in 2011-2019. Solar-Terrestrial Physics. 2020. Vol. 6. Iss. 3. P. 29-33. DOI: 10.12737/stp-63202004. 